\title{
Certificateless Public Key Cryptography: A Research Survey
}

\author{
Jacob Sayid ${ }^{1}$, Isaac Sayid ${ }^{2}$ and Jayaprakash $\operatorname{Kar}^{3}$ \\ ${ }^{1}$ Department of Information Technology \\ ${ }^{2}$ Department of Information Systems, \\ Information Security Research Group \\ Faculty of Computing \& Information Technology \\ King Abdul-Aziz University, Kingdom of Saudi Arabia, Jeddah-21589 \\ jgopabandhu@kau.edu.sa
}

\begin{abstract}
Certificateless Public Key Cryptography is a scheme that provides security by avoiding the key escrow and eliminating the drawback of identity-based cryptography. Several certificateless models have been proposed to enhance the efficiency and overcome adversaries attacks. In this paper, we survey various public key encryption schemes on certificateless setting with the security model and discuss the performance. Also, we present the generic model of Certificateless Public Key Encryption (CL-PKE) scheme proposed by various authors.
\end{abstract}

Keywords: Certificateless Cryptography, Public Key, Identity-Based Cryptography, Certificateless signatures

\section{Introduction}

Public-key encryption scheme was first come up by Rivest, Shamir and Adleman in 1978 [1]. It was based on public-key cryptography concept proposed by Diffie and Hellman in 1976 [2]. The scheme allows communicating parties to securely share a message without having previously shared-secret key. This is accomplished by generating two related keys: public-key, which is used for encryption and publicly known to others, and private-key, which is private for the user and used for decryption. However, publickey cryptography has shown some security issues. The sender has to authenticate the public key of the receiver in real world scenario. Otherwise incorrect public keys would be used to impersonate the receiver. An effective approach to provide authentication for public keys is to have a key management system called Public-Key Infrastructure. PKI is a network of trusted entities known as certificate authority (CA) that are used to authenticate the public key of the users. CAs map users' identity with their public keys and provide other key management functionalities such as certificate generations and revocations. This approach is expensive and inefficient process for any system that employs it.

\subsection{Identity-Based Cryptography}

To dispense with the need for PKI, identity-based cryptography was introduced by Shamir [3] in 1985. The idea is to eliminate the need for certificates by utilizing digitally unique information for public keys like email address. Obviously, this approach requires a trusted third party to generate private keys for its users. It is called Private Key Generator (PKG) and it distributes a master public key $m p k$ and holds the corresponding master secret key msk. For a user to obtain a private key, he/she first communicates with the PKG, which in turn uses its msk to create the private key to the user. Nevertheless, identity-based cryptography experiences the key escrow drawback which allows a PKG to 
disclose messages of its users because it has their private keys. Moreover, there are functional problems related to the key management's tasks such as key revocation [4].

\subsection{Certificateless Cryptography}

Certificateless cryptography provides security by solving the key escrow issue and avoids the problems of PKI-based schemes. Al-Riyami and Paterson [5] come out with certificateless cryptography concept in 2003. In this scheme, public keys do not require authentication. In other words, certificates and PKI are not required; instead, semi-trusted third party Key Generation Center (KGC) works to issue partial private keys $D_{I D}$ similarly

to the identity-based cryptography. To generate the actual private key, the user combines a chosen secret value with his/her $D_{I D}$ value. Hence, the key escrow drawback is resolved in certificateless cryptography and the actual private key is no longer stored in the KGC.

The upcoming sections of this paper are structured as follows. In Section 2, we define the acronyms and notations used in the whole paper. In Section 3, we present the general definition of the certificateless schemes. Next, we describe the security models and details of some well-known certificateless schemes. Next, we survey and analysis some of the well-known certificateless schemes. Finally, we conclude the survey by comparisons of the certificateless schemes performance and security.

\section{Preliminaries}

Certificateless cryptography is a public-key scheme that provides security without the need for public-keys authentication. Certificateless cryptography avoids the key escrow drawback of identity-based cryptography and replaces the public-key infrastructure by KGC. In this section, we present acronyms and notations used all over the paper. Table. 1 describes the notation. A Certificateless public-key includes three components, the user, the KGC, and a verifier. It is described by a number of probabilistic polynomial-time PPT algorithms [5]. The details of the algorithms are as follows:

- Setup: This algorithm takes security parameter $1^{k}$ and returns $m s k, m p k$ and param. It is run by KGC.

- Partial-Private-Key-Extract: The input of this algorithm includes $m s k, m p k$, param and identity ID. It returns $D_{I D}$ and the KGC run this algorithm once for each user.

- Set-Secret-Value: This algorithm receives $m p k$, param and ID as input and returns $X_{I D}$. It is run by the user.

- Set-Private-Key: This algorithm takes $m p k, D_{I D}$ and $X_{I D}$ as input and outputs $s k_{I D}$. It is run once by the user.

- Set-Public-Key: This algorithm takes $m p k$ and $X_{I D}$ to return $p k_{I D}$. It is run once by the user.

- Sign (Signcrypt): The input of this algorithm includes $m p k, I D, p k_{I D}$ and message. It outputs cipher text $\mathrm{C}$ or an error. It is run by the user/signer.

- Verify (Unsigncrypt): The input of this algorithm includes $m p k$, sk $k_{I D}$ and cipher text C. It outputs the original message or an error. It is run by the user/signer.

\section{Security Models of Certificateless Cryptography}

Security models are essential in cryptography, as they are used to analyze and define the attack power of different adversaries. Moreover, security models are used to simulate the adversary's actions and ensure their goals. In certificateless cryptography, security 
models are used to theoretically prove whether a scheme is secure or not and provide estimation to possible attacks in practice. A certificateless scheme is alleged to be secure by providing a valid proof on its matching security game. A security game is a challenger where the adversaries try to submit queries to win this game if described conditions are met. Officially, in certificateless cryptography, the adversaries are categorized as Type I and II adversaries [5][6]. Following subsections provide definition and information of each type.

\subsection{Adversary Types}

A secure certificateless scheme should counter both Type I and Type II adversaries. A Type I adversary $A_{1}$ is an outsider, not KGC nor legal user, who has the ability to selectively modify public keys of any user and may be able to get some partial private-key values but cannot disclose $m s k$ value. In other hand, a Type II adversary $A_{2}$ is a KGC which is able to view $m s k$ value but cannot change public keys of any user[24][25].

\subsection{Type I and Type II Attack Variations}

There have been different attempts to specifically describe the actions involved for adversary $A_{1}$ as well as $A_{2}$ in the security model. In general, a certificateless encryption scheme is considered secure with variations of Type I adversaries if every PPT attacker has minimal advantage in winning the indistinguishable chosen ciphertext attack 2 game. A CL scheme would be secure against Type II attack variations, if every PPT attacker, with auxiliary information equals the master private key of KGC, has minimal advantage to win the INDCCA2 game. Table 3 summarizes the attack variations for Type I and Type II [5][21].

\subsection{Adversary Power and Goals}

We first define weakest adversary goals and strongest power [7][22]

- Existential forgery goal: when the adversary has the ability to generate at least one correct signature for a message that has not been signed before.

- Strong forgery goal: when the adversary has the ability to generate at least one correct but distinct signature for a message that has been signed before.

- Adaptively chosen-message attack power: when the adversary has the ability to select signed messages adaptively in the course of attack, where the signer can be used to sign messages while the attack time. 
Table 1. Notation

\begin{tabular}{cccc}
\hline NOTATION & MEANING & NOTATION & MEANING \\
$M P K$ & MASTER PUBLIC KEY & $A_{1}$ & TYPE I ADVERSARY \\
& MASTER SECRET & $A_{2}$ & TYPE II ADVERSARY \\
$\boldsymbol{D}_{I D}$ & (PRIVATE) KEY & & \\
$\boldsymbol{p} \boldsymbol{k}_{I D}$ & PARTIAL PRIVATE KEY & PARAM & SYSTEM PARAMETER \\
$\boldsymbol{s} \boldsymbol{k}_{I D}$ & ACTUR PUBLIC KEY & $P P T$ & PROBABILISTIC, POLYNOMIAL-TIME \\
& & IND-CCA & INDISTINGUISHABLE CHOSEN \\
$\boldsymbol{x}_{I D}$ & USER SECRET VALUE & IND-CPA & CIPHERTEXT ATTACK \\
ID & USER ISTINGUISHABLE CHOSEN \\
KGC & KEY GENERATION & & PLAINTEXT ATTACK \\
& CENTER & & \\
PKG & PRIVATE KEY & & \\
\hline
\end{tabular}

Table 2. Different Variations of Type I and Type II Adversaries

\begin{tabular}{lll}
\hline NAME & NAME & NAME \\
STRONG TYPE I & WEAK TYPE IB & WEAK TYPE II \\
WEAK TYPE IA & WEAK TYPE IC & STRONG TYPE II \\
\hline
\end{tabular}

Combining the previous weak adversary goals and strongest adversary power, a secure certificateless scheme is treated as (1) existentially or (2) strong existentially unforgeable under adaptively chosen-message attacks. For the first scenario, the adversary goal is to get a faked signature $\sigma^{*}$ using identity $I D^{*}$ and message $m^{*}$ under the following circumstances:

1. $\sigma^{*}$ is valid signature for identity $I D^{*}$ and message $m^{*}$

2. $\left(I D^{*}{ }^{*} m^{*}\right)$ has not been sent to the signing oracle any time before

3. $\sigma^{*}$ has never been outputted.

Moreover, for the second scenario strong unforgeability, the conditions are the same except for the second one:

2. $\left(I D^{*}{ }_{a} m^{*}\right)$ has been sent before

\subsection{Adversaries and Oracles}

Oracles are used as responses to the adversaries' requests in the security model. There are five kinds of oracles in certificateless cryptography [8], and the access for each one is based on the security game specifications. Table 3 describes the oracles 
Table 3. Adversaries and Oracles

\begin{tabular}{|c|c|c|}
\hline ORACLE & INPUT & OUTPUT \\
\hline CREATE-USER & ID & $\begin{array}{c}\text { IF ID HAS NOT BEEN CONSTRUCTED } \\
\text { BEFORE, THE ORACLE EXECUTES THE } \\
\text { FOLLOWING AND ADDS THE RESULTS OF } \\
\text { EACH TO A LIST L. } \\
\begin{array}{c}D_{I D} \leftarrow \text { PARTIAL-PRIVATE-KEY-EXTRACT } \\
x_{I D} \leftarrow \text { SET-SECRET-VALUE } \\
p k_{I D} \leftarrow \text { SET-PUBLIC-KEY }\end{array}\end{array}$ \\
\hline PUBLIC-KEY-REPLACE & $\begin{array}{r}1-\mathrm{ID} \\
2-p k_{I D}^{s}\end{array}$ & $\begin{array}{c}\text {-THE USER ID'S PUBLIC KEY IS REPLACED } \\
\text { WITH } p k_{I D}^{s} \\
\text {-UPDATES THE RELATED RECORD IN THE } \\
\text { LIST L }\end{array}$ \\
\hline SECRET-VALUE-EXTRACT & ID & SECRET VALUE $\boldsymbol{X}_{I D}$ \\
\hline $\begin{array}{l}\text { PARTIAL-PRIVATE-KEY- } \\
\text { EXTRACT }\end{array}$ & ID & $\begin{array}{c}\text { IF ID HAS BEEN CONSTRUCTED BEFORE, } \\
\text { THIS EXTRACTS } \\
\boldsymbol{D}_{I D} \text { FROM LIST L }\end{array}$ \\
\hline SIGN (SIGNCRYPT) & $\begin{array}{r}1-M \\
2-I D \\
3-p k_{I D} \\
\end{array}$ & $\begin{array}{c}\text { SIGNATURE } \sigma \leftarrow \text { IF ID HAS BEEN } \\
\text { CONSTRUCTED BEFORE }\end{array}$ \\
\hline
\end{tabular}

\section{Survey of Proposed Protocols}

In this section, we review a number of proposed schemes for certificateless public key encryption. For each scheme, we describe the algorithms, the security model and the performance. There are two types of CL-PKE scheme constructions: concrete and generic. Concrete construction of scheme is a distinct and fully described scheme while the generic one is based on and derived from other primitives.

\subsection{Al-Riyami 1/Yum-Lee version CL-PKE [9]}

The first scheme for (CL-PKE) was proposed by [5]. He defined nomenclature, basic notations and the first framework for CL-PKE. This scheme is considered generic and is based on PKE scheme and identity-based one. The scheme is built using elliptic curve pairings.

\section{Algorithms:}

The algorithm of the scheme comprises the following:

- Initialization setup: CL-PKE scheme is initiated by KGC. This process is done once per user and outputs $m p k$ and $m s k$. The security parameter is the input for the process.

- Partial private key generation: This process is executed again by KGC and once per user. It takes the output of initialization setup as input as well as the identity of the user to construct a partial private key belongs to the user.

- Secret value setup: The user run this process taking $m p k$ and ID as input to generate a secret value that is required as input for other processes.

- Private key: This process generates a private key by taking $\left(D_{I D}, m p k, X_{I D}\right)$ as input. The generated private key is going to be known only for the generator. 
- Public key: This process generates public key by taking as input the $X_{I D}$ of the user and the $m p k$ that is extracted in the initial setup by KGC. This is done by the user.

- Encrypt: The input for encryption process is the $m p k$, user's ID, $p k_{I D}$ and the message. It produces as an output an encrypted message that can be decrypted only by user. Note that in this version, the encrypted message is produced by first applying encryption on the message via public key scheme and then via identity scheme.

- Decrypt: The input for this process is $s k_{I D}$ value of the user, $m p k$ and the encrypted message, this process can decrypt and output the original decrypted message.

\section{Security Model:}

For type I model, the scheme uses Weak Type 1b model. For type II model, the scheme uses Weak Type II model. Note that partial private key process is not accessible by the attacker. This scheme was proven insecure by [10][11]

\subsection{Al-Riyami 2 CL-PKE}

A second version of the first scheme is proposed by [2] also. Again, the scheme is considered generic and is based on PKE scheme and Identity-based one. The scheme is built using elliptic curve pairings.

\section{Algorithms:}

The algorithm of the second version of the proposed scheme is almost similar to the first version. However, it's different in the following elements:

1- Encrypt: The input for encryption process is the $m p k$, user's ID, $p k_{I D}$ and the message. It returns an encrypted message that can be decrypted only by user. Note that in Al-Riyami 2 version, the encrypted message is produced by first applying encryption on the message via identity scheme and then via public key scheme.

2- Decrypt: The decryption process in this scheme is almost identical to version 1 . However, the only difference is going to be in the order of underlying operations due to the change in encryption process.

\section{Performance}

With regard to encryption process compared to other variations of this scheme, the process is done sequentially which is not efficient.

\section{Security Model}

This version of the scheme has no proof of $A_{1}$ and $A_{2}$ security models. This scheme was proven insecure by [9]

\subsection{Al-Riyami 3 CL-PKE}

A third version of the first scheme is presented by Al-Riyami. The scheme is of generic type since it is based on -PKE scheme and identity-based one. The scheme is built using elliptic curve pairings. 


\section{Algorithms:}

The algorithm of the third version of the proposed scheme is almost similar to the first and second versions. However, it's different in the following elements:

- Encrypt: The input for encryption process is the $m p k$, user's ID, $p k_{I D}$ and the both shares $\mathrm{s} 1$ and $\mathrm{s} 2$ of the message to be encrypted $\mathrm{m}$. Where $\mathrm{s} 1+\mathrm{s} 2=\mathrm{m}$. It outputs an encrypted message that can be decrypted only by user. Note that in Al-Riyami 3 , the encrypted message is formed by applying parallel encryption on the shares of the message via identity scheme encryption and the other share via public key scheme.

- Decrypt: The decryption process in this scheme is going to be different from the other two versions since the message is split into two shares and each share is encrypted in by different schemes.

\section{Performance:}

With regard to both encryption and decryption processes compared to other variations of this scheme, the process is done in parallel which is more efficient.

\section{Security Model:}

This version of the scheme has no proof of $A_{1}$ and $A_{2}$ security models. This scheme was proven insecure by[11].

\subsection{Al-Riyami-Paterson 1 CL-PKE [5]}

A concrete type scheme is presented by Al-Riyami-Paterson. The scheme is considered concrete because it is not based on primitives like identity to compute public key. The authors adapted Fujisaki-Okamoto padding technique [12] to the scheme in order to secure it against chosen cipher text attacks.

\section{Algorithms:}

The algorithms of the Al-Riyami-Paterson 1 scheme matches the basic CL-PKE scheme pointed to earlier in several elements. These elements include partial private key generation, Secret value setup, private and public keys generation. However, it's different in the following elements:

- Initialization setup: The only difference in this process is that on top of what parameters required for this process in basic CL-PKE; there are two additional cryptographic hash functions needed.

- Encrypt and Decrypt: The encryption and decryption processes in this scheme involve more steps to be applied including checking parameters and the public key structure.

\section{Performance}

The scheme includes more underlying operations in its framework. A single encryption process requires three pairing calculations. Therefore, it can be roughly concluded that it is less efficient than the previous generic schemes and also less efficient than the second Al-Riyami-Paterson 2 scheme. 


\section{Security Model}

In random security model, the scheme's security was proven. The scheme uses Strong Type I for type I model and Weak Type II for type II model.

\subsection{Al-Riyami-Paterson 2 CL-PKE [13]}

\section{Details}

Two years after Al-Riyami-Paterson 1 scheme, the authors have proposed new generic type scheme that is based on identity-based and PKE scheme. The scheme is proposed to be more efficient than the first Al-Riyami-Patersion 1 scheme and more secure.

\section{Algorithms \\ Al-Riyami-Paterson 2 scheme is a result of optimizing double encryption construction that consists of both identity based encryption of [14] and ElGamal PKE scheme [15] as the main components or building blocks of the scheme. The scheme algorithm comprises the following:}

- Initialization setup: The input is security parameter to generate several outputs as groups to extract spaces of allowed values for message and other parameters. Moreover, a master key is generated in this process.

- Partial private key generation: The input for this process is the user's ID and generates the $D_{I D}$.

- Secret value setup: The input for this process is the output of initialization setup as well as the ID to generate a secret value.

- Private key: It is generated by taking as input the generated $X_{I D}$, output of initialization setup and entity's $D_{I D}$.

- Public key: It is generated using the exact same inputs of private key except the $D_{I D}$.

- Encrypt and Decrypt: The encryption processes in this scheme involves single pair computation per encryption which is more efficient than the Al-RiyamiPatersion 1 scheme. However, the cost of decryption is similar in both versions of the scheme. The input of encryption is the user identity and the public key. For decryption, private key is needed as input for the process.

\section{Performance}

The second scheme of Al-Riyami-Paterson 2 is more efficient than the first one since single pair computation is needed for encryption process. However, the decryption process costs are similar in both versions of the scheme.

\section{Security Model}

In random security model, the scheme's security was proven by the authors. The scheme uses Strong Type I for type I model and Weak Type II for type II model. Nevertheless, the security of this scheme is broken by [11][16][6] 


\subsection{Baek, Safavi-Naini and Susilo CL-PKE scheme}

An improved scheme that doesn't use elliptic curve pairings in its underlying architecture was proposed in [17]. The scheme is considered a modified formulation of Al-Riyami-Paterson scheme. One of the main distinct parts in this scheme is that the public key cannot be computed before partial private key computation. This has a disadvantage of preventing future encryption of messages with a public key before the private key is obtained. In contrast, the scheme with such a formulation prevents "denial of decryption".

\section{Algorithms}

Baek scheme has five elements in its algorithms instead of seven. The reason is merging of three elements in Al-Riyami scheme into one element. In other words, private key, public key generation and secret value setup are combined into one element called user key generation. The algorithm of the suggested scheme comprises the following:

- Initialization setup: This process is similar to (Al-Riyami 1) initialization setup. It is executed by KGC taking security parameter as input and generating $m s k, m p k$ and other parameters. Moreover, a master key is generated in this process.

- Partial private key generation: Same as (Al-Riyami 1) partial private key generation process. The input for this process is the user ID, msk and $m p k$ to generate $D_{I D}$.

- Key Generation: A process that combines multiple processes in (Al-Riyami 1) scheme. The input for this process is the $D_{I D}$ and the user identity to generate public and private key for users.

- Encrypt: Exactly as encryption process in (Al-Riaymi 1) scheme. Takes the $p k_{I D}$, $m p k$ and ID as input to generate an encrypted message.

- Decrypt: Different than decryption process in (Al-Riaymi 1) scheme. Takes the user private key and $m p k$ as input to generate a decrypted message.

\section{Performance}

Compared to (Al-Riyami 1) scheme, this scheme is more efficient since it combines multiple processes of key generation into single process.

\section{Security Model}

Obviously, the scheme doesn't rely on secret value which makes Weak Type Ia security model improper. In random security model, the scheme's security was proven by the authors. The scheme uses Strong Type I for type I model and Weak Type II for type II model.

\subsection{Libert-Quisquater CL-PKE scheme (concrete version)}

An efficient concrete CL-PKE scheme was proposed by [11]. The scheme seems to be a newer version of Al-Riyami-Paterson scheme since the author called it "NewFullCLE". The scheme can be viewed as identity based encryption combined with ElGamal encryption. 


\begin{abstract}
Algorithms
Libert-Quisquater scheme has seven elements in its algorithm and it is highly similar to Al-Riyami-Paterson 2 scheme elements with little differences in some underlying operations. The elements in this scheme are initialization setup, partial private key generation, secret value setup, private key generation, public key generation, encrypt and decrypt
\end{abstract}

\title{
Performance
}

The scheme is comparable to Baek Scheme as the author claimed. It is more efficient than similar schemes proposed such as Shi and $\mathrm{Li}$ [18].

\section{Security Model}

In random security model, the scheme's security was proven by the authors. The scheme uses Strong Type I for type I model and Weak Type II for type II model.

\subsection{Libert-Quisquater CL-PKE scheme (generic version 1)}

Libert-Quisquater [11] proposed secured version of Al-Riyami 1 scheme. The proposed scheme applied Fujisaki-Okamoto transformation. This technique is a modified version of the Fujisaki-Ocamoto conversion which converts IND-CPA attacks into INDCCA ones. The modification is done by including the user/receiver ID including the public key within the input of hash functions used in the scheme algorithms.

\section{Algorithms}

This scheme is almost identical to AL-Riyami 1 scheme in terms of efficiency and how the algorithms are executed except two of them. These two algorithms are the encryption and decryption. User identity is included in the hash functions used for both encryption and decryption.

\section{Performance}

The scheme is similar Al-Riyami 1 scheme since it extends it. The encryption process compared to other generic versions of Libert-Quiquater is considered less efficient.

\section{Security Model}

In random security model, the scheme uses Strong Type I for type I model and Weak Type II for type II model.

\subsection{Libert-Quisquater CL-PKE scheme (generic version 2)}

Libert-Quisquater [11] proposed secured version of Al-Riyami 2 scheme. The proposed scheme also applied Fujisaki-Okamoto transformation. As mentioned earlier, the technique is a modified version of the Fujisaki-Ocamoto conversion which converts IND-CPA attacks into IND-CCA attacks. The modification is done by including the user/receiver ID including the public key within the input of hash functions used in the scheme algorithms.

\section{Algorithms}

This scheme is similar to AL-Riyami 2 scheme in terms of efficiency and how the algorithm is executed except two steps which are the encryption and decryption. User identity is included in the hash functions and used for both encryption and decryption. 


\section{Performance}

The scheme is similar Al-Riyami 2 scheme since it extends it. The encryption process compared to other generic versions of Libert-Quiquater is considered less efficient.

\section{Security Model}

In random security model, the scheme uses Strong Type I for type I model and Weak Type II for type II model.

\subsection{Libert-Quisquater CL-PKE scheme (generic version 3)}

Libert-Quisquater [11] proposed secured version of Al-Riyami 2 scheme. The proposed scheme also applied Fujisaki-Okamoto transformation. The transformation is done by including the user/receiver ID including the public key within the input of hash functions used in the scheme algorithms.

\section{Algorithms}

This scheme is similar to AL-Riyami 3 scheme in terms of efficiency and how the algorithms are executed except two elements. These two elements are the encryption and decryption. User identity is included in the hash functions used for both encryption and decryption.

\section{Performance}

The scheme is similar Al-Riyami 3 scheme since it extends it. The encryption process compared to other generic versions of Libert-Quiquater is considered more efficient since parallelism is exploited.

\section{Security Model}

In random security model, the scheme uses Strong Type I for type I model and Weak Type II for type II model.

\subsection{Scheme 11: Dent, Libert and Paterson (concrete version)}

Dent, Libert and Paterson [19] proposed concrete CL-PKE scheme that is secure and resist both Strong type I and II attacks. The scheme doesn't require random oracle model. The scheme uses bilinear map groups in its algorithms. It is an extended version of Boyen, Mei and Waters [20] scheme and applies its concepts.

\section{Algorithms:}

The algorithm of this scheme comprises the following:

- Initialization setup: Using bilinear map groups, this process generate both $m p k$ and $m s k$ for KGC.

- Partial private key generation: The input of this process is ID, $m p k$ and a random value generated in initialization setup process to generate the $D_{I D}$.

- Secret value setup: This process generates a random value as the secret value $X_{I D}$ taking as input mpk.

- Private key: It is generated by taking as input the generated $X_{I D}, D_{I D}$ and $m p k$.

- Public key: It is generated taking as input the $m p k$ and user $X_{I D}$. 
- Encrypt: The encryption process in this scheme takes the message, user's public key, user's ID and $m p k$ to generate an encrypted message.

- Decrypt: The decryption process in this scheme takes the encrypted message, user's private key and $m p k$ to generate decrypted message.

\section{Performance}

The author claimed that the public keys and encrypted messages are relative short which make the scheme perform better.

\section{Security Model}

The scheme doesn't require random oracle model. It uses Strong Type I for type I model and Strong Type II for type II model.

Table 4. Comparison of Relative Performance between Proposed Schemes

\begin{tabular}{ccc}
\hline SCHEME 1 & RELATIVE PERFORMANCE & SCHEME 2 \\
& COMPARISON & \\
AL-RIYAMI 1/YUM-LEE & IS ALMOST EQUAL TO & AL-RIYAMI 2 \\
AL-RIYAMI 1/YUM-LEE & IS LESS EFFICIENT THAN & AL-RIYAMI 3 \\
AL-RIYAMI 2 & IS LESS EFFICIENT THAN & AL-RIYAMI 3 \\
AL-RIYAMI-PATERSON 1 & IS LESS EFFICIENT THAN & AL-RIYAMI-PATERSON 1 \\
AL-RIYAMI 1/YUM-LEE & IS LESS EFFICIENT THAN & BAEK, SAFAVI-NAINI AND \\
LIBERT-QUISQUATER & IS MORE EFFICIENT THAN & SUSILO \\
(CONCRETE) & & SHI AND LI \\
LIBERT-QUISQUATER & IS ALMOST EQUAL TO & LIBERT-QUISQUATER \\
(GENERIC 1) & & (GENERIC 2) \\
LIBERT-QUISQUATER & IS LESS EFFICIENT THAN & LIBERT-QUISQUATER \\
(GENERIC 1) & & (GENERIC 3) \\
LIBERT-QUISQUATER & IS LESS EFFICIENT THAN & LIBERT-QUISQUATER \\
(GENERIC 2) & & (GENERIC 3) \\
\hline
\end{tabular}


Table 5. Comparison of Certificateless Cryptography Schemes

\begin{tabular}{|c|c|c|c|c|}
\hline SCHEME & $\begin{array}{l}\text { TYPE OF } \\
\text { SCHEME }\end{array}$ & $\begin{array}{l}\text { TYPE I } \\
\text { SECURITY } \\
\text { MODEL }\end{array}$ & $\begin{array}{l}\text { TYPE II } \\
\text { SECURITY } \\
\text { MODEL }\end{array}$ & SECURE? \\
\hline $\begin{array}{l}\text { AL-RIYAMI 1/YUM- } \\
\text { LEE [9][21] }\end{array}$ & GENERIC & WEAK TYPE IB & WEAK TYPE II & No [10][16] \\
\hline AL-RIYAMI 2 [9] & GENERIC & $\begin{array}{l}\text { NO PROOF } \\
\text { AVAILABLE }\end{array}$ & $\begin{array}{l}\text { NO PROOF } \\
\text { AVAILABLE }\end{array}$ & No \\
\hline AL-RIYAMI 3 [9] & GENERIC & $\begin{array}{c}\text { NO PROOF } \\
\text { AVAILABLE }\end{array}$ & $\begin{array}{l}\text { NO PROOF } \\
\text { AVAILABLE }\end{array}$ & No [16] \\
\hline $\begin{array}{c}\text { AL-RIYAMI- } \\
\text { PATERSON } 1 \text { [5][9] }\end{array}$ & CONCRETE & STRONG TYPE I & WEAK TYPE II & YES \\
\hline $\begin{array}{c}\text { AL-RIYAMI- } \\
\text { PATERSON } 2 \text { [9][13] }\end{array}$ & GENERIC & STRONG TYPE I & WEAK TYPE II & $\mathrm{No}[11][16][6]$ \\
\hline $\begin{array}{c}\text { BAEK, SAFAVI- } \\
\text { NAINI AND SUSILO } \\
\text { CERTIFICATELESS } \\
\end{array}$ & CONCRETE & STRONG TYPI & WEAK TYPE II & YES \\
\hline $\begin{array}{c}\text { LIBERT- } \\
\text { QUISQUATER } \\
\text { (CONCRETE) }\end{array}$ & CONCRETE & STRONG TYPI & WEAK TYPE II & YES \\
\hline $\begin{array}{c}\text { LIBERT- } \\
\text { QUISQUATER } \\
\text { (GENERIC } 1 \text { ) }\end{array}$ & GENERIC & STRONG TYPI & WEAK TYPE II & YES \\
\hline $\begin{array}{c}\text { LIBERT- } \\
\text { QUISQUATER } \\
\text { (GENERIC 2) }\end{array}$ & GENERIC & STRONG TYPI & WEAK TYPE II & YES \\
\hline $\begin{array}{c}\text { LIBERT- } \\
\text { QUISQUATER } \\
\text { (GENERIC 3) }\end{array}$ & GENERIC & STRONG TYPI & WEAK TYPE II & YES \\
\hline $\begin{array}{c}\text { DENT, LIBERT AND } \\
\text { PATERSON }\end{array}$ & CONCRETE & STRONG TYPI & STRONG TYPE & YES \\
\hline
\end{tabular}

\section{Conclusion}

In this paper, we have presented all version of CL-PKE schemes proposed by by AlRiyami, Baek, et al and Libert-Quisquater, We have given a comparative study on performance and security of the proposed scheme. From this survey, we can identify the drawbacks of various CL-PKE schemes. This help to construct an efficient and secure scheme that reduces the vulnerability.

\section{Acknowledgements}

We would like to thank to our supervisor Dr. Jayaprakash Kar for his valuable suggestions and comments that helped improving this work. This support is greatly appreciated.

\section{References}

[1] R. L. Rivest, A. Shamir and L. Adleman, "A Method for Obtaining Digital Signatures and Public-key Cryptosystems", Commun ACM, vol. 21, no. 2, (1978) Feb., pp. 120-126.

[2] W. Diffie and M. Hellman, "New Directions in Cryptography", IEEE Trans Inf Theor, vol. 22, no. 6, (1976), pp. 644-654.

[3] A. Shamir, "Identity-Based Cryptosystems and Signature Schemes", in Advances in Cryptology, G. R. Blakley and D. Chaum, Eds. Springer Berlin Heidelberg, (1984), pp. 47-53. 
[4] A. W. Dent, "A survey of certificateless encryption schemes and security models", Int. J. Inf. Secur., vol. 7, no. 5, (2008) May, pp. 349-377.

[5] S. S. Al-Riyami and K. G. Paterson, "Certificateless Public Key Cryptography", in Advances in Cryptology - ASIACRYPT 2003, C.-S. Laih, Ed. Springer Berlin Heidelberg, (2003), pp. 452-473.

[6] Z. Zhang, D. S. Wong, J. Xu and D. Feng, "Certificateless Public-Key Signature: Security Model and Efficient Construction", in Applied Cryptography and Network Security, J. Zhou, M. Yung, and F. Bao, Eds. Springer Berlin Heidelberg, (2006), pp. 293-308.

[7] S. Goldwasser, S. Micali and R. L. Rivest, "A Digital Signature Scheme Secure Against Adaptive Chosen-message Attacks", SIAM J Comput, vol. 17, no. 2, (1988) Apr., pp. 281-308.

[8] X. Huang, Y. Mu, W. Susilo, D. S. Wong and W. Wu, "Certificateless Signature Revisited", in Information Security and Privacy, J. Pieprzyk, H. Ghodosi, and E. Dawson, Eds. Springer Berlin Heidelberg, (2007), pp. 308-322.

[9] S. Al-Riyami, Cryptographic schemes based on elliptic curve pairings. University of London, (2004).

[10] D. Galindo, P. Morillo and C. Ràfols, "Breaking Yum and Lee Generic Constructions of CertificateLess and Certificate-Based Encryption Schemes", in Public Key Infrastructure, A. S. Atzeni and A. Lioy, Eds. Springer Berlin Heidelberg, (2006), pp. 81-91.

[11] B. Libert and J.-J. Quisquater, "On Constructing Certificateless Cryptosystems from Identity Based Encryption”, in Public Key Cryptography - PKC 2006, M. Yung, Y. Dodis, A. Kiayias, and T. Malkin, Eds. Springer Berlin Heidelberg, (2006), pp. 474-490.

[12] E. Fujisaki and T. Okamoto, "Secure integration of asymmetric and symmetric encryption schemes", in Crypto, vol. 99, (1999), pp. 537-554.

[13] S. S. Al-Riyami and K. G. Paterson, "CBE from CL-PKE: A Generic Construction and Efficient Schemes”, in Public Key Cryptography - PKC 2005, S. Vaudenay, Ed. Springer Berlin Heidelberg, (2005), pp. 398-415.

[14] D. Boneh and M. Franklin, "Identity-Based Encryption from the Weil Pairing", in Advances in Cryptology - CRYPTO 2001, J. Kilian, Ed. Springer Berlin Heidelberg, (2001), pp. 213-229.

[15] T. ElGamal, "A Public Key Cryptosystem and a Signature Scheme Based on Discrete Logarithms", in Advances in Cryptology, G. R. Blakley and D. Chaum, Eds. Springer Berlin Heidelberg, (1984), pp. 1018.

[16] Z. Zhang and D. Feng, "On the security of a certificateless public-key encryption (2005)", Available Fro M Httpeprint Iacr Org2005426 Proof Lemma, vol. 2.

[17] J. Baek, R. Safavi-Naini and W. Susilo, "Certificateless Public Key Encryption Without Pairing", in Information Security, J. Zhou, J. Lopez, R. H. Deng, and F. Bao, Eds. Springer Berlin Heidelberg, (2005), pp. 134-148.

[18] Y. Shi and J. Li, "Provable Efficient Certificateless Public Key Encryption", IACR Cryptol. EPrint Arch., vol. 2005, (2005), p. 287.

[19] A. W. Dent, B. Libert and K. G. Paterson, "Certificateless encryption schemes strongly secure in the standard model”, in Public Key Cryptography-PKC 2008, Springer, (2008), pp. 344-359.

[20] X. Boyen, Q. Mei and B. Waters, "Direct chosen ciphertext security from identity-based techniques", in Proceedings of the 12th ACM conference on Computer and communications security, (2005), pp. 320329.

[21] J. Kar, "Provably Secure Identity-Based Aggregate Signcryption Scheme in Random Oracles", International Journal of Network Security, Taiwan, vol. 17, no.5, (2015), pp. 580-587.

[22] J. Kar, "Deniable Authentication Protocol based on Discrete Logarithms and Integer Factorization Problems", ICIC Express Letters, Japan, 2012. vol. 7, no. 7, (2013) July, pp. 2061-2067.

[23] D. H. Yum and P. J. Lee, "Generic Construction of Certificateless Encryption", in Computational Science and Its Applications - ICCSA 2004, A. Laganá, M. L. Gavrilova, V. Kumar, Y. Mun, C. J. K. Tan, and O. Gervasi, Eds. Springer Berlin Heidelberg, (2004), pp. 802-811.

[24] J.Kar, "Non-interactive Deniable Authentication Protocol using generalized ECDSA Signature Scheme”, International Journal of Smart Home. Korea, vol. 5, no. 4, (2011) Oct., pp. 39-49.

[25] J. Kar \& D. M. Alghazzawi, "On Construction of Signcryption Scheme for Smart Card Security", IEEE International Conference on Intelligence and Security Informatics (IEEE ISI 2015), US, (2015), pp. 109-113.

\begin{abstract}
Authors
Yaqoob S. Ikram has completed bachelor of science (IT) and pursuing M.S in Information Technology in Faculty of Computing \& Information Technology, King Abdul-Aziz University, Kingdom of Saudi Arabia, in 2013. His research interests include cloud security, host-based intrusion detection systems and improved agile models for software development. Mr.Yaqoob worked as a developer in different government project at Kingdom of Saudi Arabia and other countries.
\end{abstract}


Isaac Sayid has completed bachelor of science (IT) and pursuing M.S in Information Technology in Faculty of Computing \& Information Technology, King Abdul-Aziz University, Kingdom of Saudi Arabia, in 2013. His current research interests include Instrustion detection and Prevention system and Cryptography.

Jayaprakash Kar has received his M.Sc and M.Phil in Mathematics from Sambalpur University, M.Tech and Ph.D in Computer Science (Cryptographic Protocols) from Utkal University, India. Currently he is working as Assistant Professor in the Department of Information Systems, Faculty of Computing and Information Technology. He is actively associated with Information Security Research Group, King Abdulaziz University, Saudi Arabia. His current research interests is in development and design of provably secure cryptographic protocols and primitives using Elliptic Curve and Pairing based Cryptography 
International Journal of Security and Its Applications

Vol. 10, No. 7 (2016) 